\title{
Recognising adverse drug reactions involving the respiratory system
}

\author{
A J Avery
}

Anthony J Avery

Reader in Primary Health Care, University Hospital Nottingham

Correspondence to:

Anthony Avery

Division of General

Practice

School of Community

Health Sciences

The Medical School

University Hospital

Nottingham, NG7 2UH

tony.avery@nottingham.ac.uk

Date submitted: 08/06/01

Date accepted: 10/06/01

Prim Care Respir J 2001:

10(2) 26-27
${ }^{6} \mathrm{~F}$ irst do no harm" is one of our fundamental ethical principles and so when a patient presents with a serious problem related to medicine we have prescribed this strikes to the core. Even if we have done no wrong, it still leaves a sickening feeling to realise that a person has been harmed because of something we have prescribed. ${ }^{1}$

In this edition of the journal Bhatia and colleagues [pp 39] describe the adverse drug reactions (ADRs) that can affect the respiratory system. ${ }^{2}$ In this editorial I highlight the implications of the article for prescribing in primary care and focus on issues around the recognition of ADRs.

An adverse drug reaction is defined as "an adverse outcome that occurs during or following clinical use of a drug... that is judged to be caused by the drug". ${ }^{3}$ The article by Bhatia and colleagues provides details of 19 types of drug-induced lung disease involving around 100 drugs or drug groups. Fortunately the majority of drugs mentioned are used rarely or never in primary care and few of the remaining drugs are prescribed on a daily basis by most GPs. Also, most of the adverse drug reactions listed are rare in primary care. Nevertheless, the article presents us with two particular challenges. Firstly, how do we heighten our awareness of relatively rare ADRs involving drugs that we do not use everyday? Secondly, how do we avoid the ADRs that most primary care prescribers would be expected to recognise?

One of the problems with diagnosing ADRs causing lung disease is that the presentation may be similar to that of conditions seen very commonly in primary care. For example, in younger patients presenting with cough or shortness of breath our first thoughts are of conditions such as respiratory tract infection and asthma. We may think about drugs as a cause of the symptoms, but this is usually for well-known problems such as ACE inhibitors causing cough and beta-blockers or NSAIDs causing exacerbations of asthma. For rarer conditions we are hampered by the way in which we tend to make diagnoses in primary care - a method that relies heavily on pattern recognition.

One of the strengths of general practice is the way that we can get the right diagnosis in a relatively short space of time. This is because we tend to use a hypothetico-deductive approach ${ }^{4}$ in which questions are used to either confirm or refute potential diagnoses right from the early stages of the consultation. In most cases this works well, but the technique is prone to confirmation bias whereby we may hone down too early on a diagnosis because it fits a pattern of presentation that we have seen previously. This failing is not unique to GPs and is recognised to be an important cause of human error. ${ }^{5}$
How do we combat this problem? Having a good knowledge base may help especially if this is accompanied by a questioning attitude to the diagnostic process. In particular, it is important to try to use a systematic approach to diagnosis if a patient does not seem to be responding to treatment. This is especially important when a patient has pre-existing lung disease. For example, with COPD it is easy to assume that any increase in breathlessness is due to an exacerbation of the condition and it may take several consultations before we abandon the hypothesis and consider other causes for the symptoms.

For ADRs that are relatively rare in primary care, increased diagnostic vigilance may be helpful, but it is important to consider other methods to help us recognise problems at an early stage. Systematically checking through a patient's medications along with information on side effects is one approach. Another is to make better use of our clinical computing systems. For example, most of us will be aware of clinical systems that alert us to important co-morbidities such as asthma when we are prescribing. In time there will be increasingly availability of systems that recognise symptoms such breathless and alert us if the patient is taking any drug known to cause respiratory disease. This approach could shorten the time it takes to diagnose an ADR and minimise patient morbidity. Nevertheless, it is important to recognise that systems need to be designed to give timely and appropriate feedback to GPs and not to swamp them with alert messages.

What should we do about the ADRs that are wellknown to GPs? One example is the prescribing of beta-blockers (particularly those that are non cardioselective) to patients with asthma. ${ }^{6}$ This can result in death $^{6}$ and many people would view these cases as clear examples of prescribing errors. Nevertheless, detailed analysis sometimes reveals a more complex picture. For example, some patients present with possible asthma when they have been stable on a betablocker for angina or hypertension for many years. In these cases it can take some time to change the drug while keeping the pre-existing morbidities under control. In other cases there is clear evidence of a doctor inappropriately prescribing a beta-blocker without recognising that the patient has asthma. In these cases it is clear that we need systems in place to avoid such prescribing. Computerised alerts have a potentially important role here, but it should be recognised that these alerts rely on accurate morbidity data being put on computer, ${ }^{7}$ and computer systems having the necessary alert facility. ${ }^{7}$ Other methods include vigilance by pharmacists and encouraging patients to read product information leaflets. 
Another ADR that is potentially life-threatening in primary care is bronchoconstriction caused by aspirin and other NSAIDs. ${ }^{8}$ These drugs are contraindicated in patients known to have previous sensitivity and they should be prescribed with caution in patients with asthma. The latter group should be advised to stop the drugs if there is any worsening of asthma symptoms. It is important for patients to report these ADRs so that they can be advised about avoiding NSAIDs in the future, including those that they might purchase without a prescription. Also, it is essential that information on NSAID sensitivity is displayed prominently in the medical records and incorporated into computerised alerts where these are available.

ADRs involving the respiratory system present a particular challenge because of the non-specific way in which many of them present. Nevertheless, with adequate training and effective systems in place it should be possible for us to avoid ADRs, or to pick them up as soon as possible. A high index of suspicion is needed for those drugs that we do not use commonly in primary care.

\section{REFERENCES}

1. Wu AW. Medical error: the second victim. $B M J$ 2000;320:72627.

2. Bhatia P, O'Reilly JF, Li-Kam-Wa E. Adverse drug reactions and the respiratory system. Prim Care Respir J 2001:10(2): 39-43.

3. Strom BL (Ed). Pharmacoepidemiology. Chichester: Wiley \& Sons Ltd., 2000.

4. Fraser RC. Clinical method: a general practice approach (2nd edition). Oxford: Butterworth-Heinemann, 1992.

5. Reason J. Human Error. Cambridge: Cambridge University Press, 1990.

6. Stockley I. Drug Interactions ( $5^{\text {th }}$ edition). London: Pharmaceutical Press, 1999.

7. Avery A J Computerised alerts for potential drug hazards Prescriber 2000;11(7):113-117.

8. Amadio P, Cummings DM, Amadio P. Nonsteroidal anti-inflammatory drugs: tailoring therapy to achieve results and avoid toxicity. Postgraduate Medicine 1999;93:73-97.

\title{
Choice of add-on therapy in asthma - another inhaler or a tablet / syrup? A survey of 1415 UK asthmatics
}

\author{
J Tuggey, H S R Hosker, P Chetcuti, K Brownlee, J Taylor, C MacGowan
}

\author{
ABSTRACT \\ Keywords \\ Asthma; patient compliance; anti-asthmatic agents and anti- \\ inflammatory agents \\ Objectives To determine preferences for asthma treatment given as \\ inhaled therapy or as a tablet / syrup, to identify factors that influ- \\ ence this choice, and to assess how many patients would sacrifice \\ greater efficacy of therapy in order to use the delivery route of their \\ choice. \\ Design Postal Questionnaire to patients with an active diagnosis of \\ asthma aged less than 60 years under the care of general practition- \\ ers, paediatricians or adult respiratory physicians. \\ Main Outcome Measure Patient preference for inhaled therapy or \\ tablet/syrup.
}

Results 715 replies were analysed (93\% Caucasian). 58\% (417) preferred tablets $(p<0.01)$ based on an equal chance of symptom improvement. There was no sex difference, but more children aged 6-10 and parents of under 6 year olds preferred tablets or syrup than adults $(65 \%$ vs. $54 \%, p=0.03)$. Preference for tablets increased with number of current inhalers $(\mathrm{p}<0.05)$ but there was no correlation with total number of puffs per day or numbers of existing tablets taken. $238(36 \%)$ opted for their preferred route of delivery in preference to greater efficacy.

Conclusion More asthmatics would choose a tablet or syrup than another inhaler as add-on therapy for their asthma. This preference was more marked in children and in patients already taking several inhalers. $36 \%$ of patients are prepared to sacrifice greater efficacy in favour of their choice of route of delivery.

\section{INTRODUCTION}

Compliance with regular therapy is an important issue in asthma. Many factors influence patient compliance and these need to be addressed as part of choosing the appropriate therapy for individual patients. The importance of addressing compliance is emphasised in all major guidelines, including the British Asthma guidelines.

Inhaled therapy has been the mainstay of treatment for asthma over the past two decades, as a means of achieving good local efficacy with the least systemic side effects. The recent introduction of a new class of oral therapy for asthma, the leukotriene antagonists, has raised new issues concerning patient's choice of delivery route for regular treatment. ${ }^{2}$ There have been few objective studies assessing preference with oral medications. ${ }^{3,4}$ Tettersell suggests that poor compliance with inhaled therapy is associated with a preference for tablets. ${ }^{5}$ It would seem logical that one path to improving compliance would be to offer treatment that patients prefer.

The principal aims of our study were to explore the preferences of patients with asthma regarding delivery route for their medication, and to identify factors that influenced their choice. A further aim was to assess whether patients would sacrifice greater efficacy of therapy in order to use the delivery route of their choice. 